\title{
Report
}

\section{Carbon-Based Supercapacitors Produced by Activation of Graphene}

Yanwu Zhu, ${ }^{1}$ Shanthi Murali, ${ }^{1}$ Meryl D. Stoller, ${ }^{1}$ K. J. Ganesh, ${ }^{1}$ Weiwei Cai, ${ }^{1}$ Paulo J. Ferreira, ${ }^{1}$ Adam Pirkle, ${ }^{2}$ Robert M. Wallace, ${ }^{2}$ Katie A. Cychosz, ${ }^{3}$ Matthias Thommes, ${ }^{3}$ Dong Su, ${ }^{4}$ Eric A. Stach, ${ }^{4}$ Rodney S. Ruoff $^{1 *}$

${ }^{1}$ Department of Mechanical Engineering and Materials Science and Engineering Program, University of Texas at Austin, One University Station C2200, Austin, TX 78712, USA. ${ }^{2}$ Department of Materials Science and Engineering, University of Texas at Dallas, 800 W. Campbell Rd, Richardson, TX 75080, USA. ${ }^{3}$ Quantachrome Instruments, 1900 Corporate Drive, Boynton Beach, FL 33426, USA. ${ }^{4}$ Center for Functional Nanomaterials, Brookhaven National Laboratory, Upton, NY 11973, USA.

*To whom correspondence should be addressed. E-mail: r.ruoff@mail.utexas.edu

Supercapacitors, also called ultracapacitors or electrochemical capacitors, store electrical charge on high surface area conducting materials. Their widespread use is limited by their low energy storage density and relatively high effective series resistance. Using chemical activation of exfoliated graphite oxide, we synthesized a porous carbon with a BET surface area of up to $\mathbf{3 1 0 0}$ square meters per gram, a high electrical conductivity, and a low $\mathbf{O}$ and $\mathbf{H}$ content. This $\mathbf{s p}^{2}$-bonded carbon has a continuous three-dimensional network of highly curved, atom thick walls that form primarily $0.6-5 \mathrm{~nm}$ width pores. Two-electrode supercapacitor cells constructed with this carbon yielded high values of gravimetric capacitance and energy density with organic and ionic liquid electrolytes. The processes used to make this carbon are readily scalable to industrial levels.

Supercapacitors store energy by forming a double layer of electrolyte ions on the surface of conductive electrodes.

Supercapacitors are not limited by the electrochemical charge transfer kinetics of batteries and thus can operate at very high charge and discharge rates, and can have lifetimes of over a million cycles (1). However, the energy stored in supercapacitors is currently an order of magnitude lower than batteries, which limits their adoption to those applications that require high cycle life and power density. The energy density of existing state-of-the-art supercapacitor devices, mainly based on porous activated carbon (AC), is about 4-5 $\mathrm{Wh} / \mathrm{kg}$ while that of lead acid batteries is in the range of 26$34 \mathrm{Wh} / \mathrm{kg}(2)$. A typical AC material, with a BrunauerEmmett-Teller (BET) specific surface area (SSA) in the range of $1000-2000 \mathrm{~m}^{2} / \mathrm{g}$ and a pore size distribution in the range of 2-5 nm, has a gravimetric capacitance of 100-120 F/g in organic electrolytes (3). Research has thus been focused on increasing energy density without sacrificing cycle life or high power density (4). An increase capacitance in the organic electrolyte tetraethylammonium tetrafluoroborate
(TEA $\mathrm{BF}_{4}$ ) in acetonitrile (AN) has been reported using carbide-derived carbons (CDCs) (5). Metal oxides such as $\mathrm{RuO}_{2}$ or $\mathrm{MnO}_{2}(6), \mathrm{MoO}_{3}(7)$, and electronically conducting polymers (8), or their composites, have been used to increase specific capacitance via pseudo-capacitive redox reactions. Although capacitances of up to $1300 \mathrm{~F} / \mathrm{g}$ (e.g., with $\mathrm{MnO}_{2}$ ) have been reported in aqueous electrolytes (9), the low electrical conductance, poor compatibility with organic electrolytes, and short cycle life have limited the practical application of these pseudo-capacitive materials. Carbon nanotubes (CNTs), especially single walled CNTs (SWNTs) have an ideal limit SSA of $1300 \mathrm{~m}^{2} / \mathrm{g} \mathrm{(10),} \mathrm{and} \mathrm{can} \mathrm{have} \mathrm{high}$ electrical conductance along the tubes and demonstrate good performance in organic electrolyte (11). However, the high cost for mass production of high quality SWNTs is a challenge for the commercialization of SWNT-based supercapacitors.

Graphene has a theoretical SSA of $2630 \mathrm{~m}^{2} / \mathrm{g}$ and a very high intrinsic electrical conductivity in plane, as well as high mechanical strength and chemical stability (12). Graphenebased material derived from graphite oxide (GO) is now being manufactured in ton quantities at low cost (13). Our group previously demonstrated supercapacitors based on reduced graphene oxide with capacitance values of approximately $130 \mathrm{~F} / \mathrm{g}$ and $100 \mathrm{~F} / \mathrm{g}$ in aqueous $\mathrm{KOH}$ and organic electrolytes, respectively (14). With a low equivalent series resistance (ESR), the supercapacitor performance did not show significant degradation with an increase in the scan rate. Various graphene-based materials derived from GO have reported high end capacitance values of $\sim 200 \mathrm{~F} / \mathrm{g}$ in aqueous electrolytes $(15,16), \sim 120 \mathrm{~F} / \mathrm{g}$ in organic electrolytes (16, 17 ), and $\sim 75 \mathrm{~F} / \mathrm{g}$ in an ionic liquid (18). Recently, supercapacitors using oriented graphene grown on nickel by chemical vapor deposition were reported (19) that demonstrated efficient filtering of $120 \mathrm{~Hz}$ current with an RC time constant of less than $0.2 \mathrm{~ms}$, but at the cost of effective 
energy storage due to the very low density of the electrode material.

To date, the reported SSA values of carbon materials derived from GO have been well below $2630 \mathrm{~m}^{2} / \mathrm{g}$. Here, we report a simple activation with $\mathrm{KOH}$ of microwave exfoliated GO (MEGO) and thermally exfoliated GO (TEGO), to achieve SSA values up to $3100 \mathrm{~m}^{2} / \mathrm{g}$. As described in (20), we prepared MEGO powders by irradiating $\mathrm{GO}$ in a microwave oven. The as-made MEGO powder was then placed in $\mathrm{KOH}$ solution, followed by filtration and drying, to form a series of $\mathrm{MEGO} / \mathrm{KOH}$ mixtures for chemical activation. Each $\mathrm{MEGO} / \mathrm{KOH}$ mixture was put in a tube furnace under flowing argon at a pressure of about 400 Torr, and heated at $800{ }^{\circ} \mathrm{C}$ for one hour. Figure $1 \mathrm{~A}$ shows a schematic of this activation process. Chemical activation has been extensively used to obtain porous ACs (21). $\mathrm{KOH}$ activation has been used on CNTs (22), carbon nanofibers (23), and polyacrylonitrile-carbon nanotubes composites (24), and improved porosity and enhanced supercapacitor performance was reported. It is suggested that the activation of carbon with $\mathrm{KOH}$ proceeds (25) as, $6 \mathrm{KOH}+\mathrm{C} \leftrightarrow 2 \mathrm{~K}+$ $3 \mathrm{H}_{2}+2 \mathrm{~K}_{2} \mathrm{CO}_{3}$, followed by decomposition of $\mathrm{K}_{2} \mathrm{CO}_{3}$ and/or reaction of $\mathrm{K} / \mathrm{K}_{2} \mathrm{CO}_{3} / \mathrm{CO}_{2}$, with carbon.

The activation with $\mathrm{KOH}$ generated nanoscale pores in the product carbon. The SSA of the a-MEGO could be readily controlled by the ratio of $\mathrm{KOH}$ versus $\mathrm{MEGO}$ (fig. S1). The scanning electron microscopy (SEM) image in Fig. 1 B shows the microstructure of an a-MEGO chunk. Figure 1, C to F, shows high-resolution SEM (C) annular dark field scanning transmission electron microscopy (ADF-STEM) (D) and high-resolution TEM (HR-TEM) (E-F) images of the microstructure. These images (and additional images presented as fig. S2 and movie S1) clearly indicate that the activation process etches the MEGO and has generated a three-dimensional (3D) distribution of what are referred to as mesopores in the porous materials literature. Remarkably, the activation with $\mathrm{KOH}$ yields a continuous $3 \mathrm{D}$ network of pores of extremely small size, ranging from $<1 \mathrm{~nm}$ to $10 \mathrm{~nm}$. Thus, it appears that the chemical activation is not merely digesting the MEGO but is also dramatically restructuring it. (The 3D nature of these very small pores makes a statistically accurate quantitative analysis of the distribution of pores sizes by electron microscopy difficult, as not all pores are visible in a given image.) The spherical aberration corrected HR-TEM image presented as Fig. 1E (taken at $80 \mathrm{kV}$ to prevent electron beam damage) further corroborates the presence of a dense pore structure, and indicates that the walls of the pores are composed of highly curved carbon sheets, of predominately single layer thickness. An exit wave reconstructed image (Fig. 1F) taken with the TEAM instrument (spherical and chromatic aberration correction, at $80 \mathrm{kV}$ ) clearly resolves the individual carbon atoms in the structure. This image shows that a-MEGO is composed of nmembered rings in plane, with $\mathrm{n}$ varying between 5 and 8 . Additionally, it is clear that even as the sheets bend through high degrees of curvature, the in-plane crystallinity is preserved.

Electron paramagnetic resonance (EPR) data (fig. S3) indicate an unpaired spin count at the ppm level. This-in hand with the very low $\mathrm{H}$ and $\mathrm{O}$ content in the a-MEGO by elemental analysis - show that a-MEGO has a small fraction of edge atoms. (If a significant fraction of the $\mathrm{C}$ were edge atoms they would either be functionalized or would be present as "dangling bonds" which would register an EPR signal).

Figure 2, A and B, shows characterization of a sample of a-MEGO by synchrotron powder X-ray diffraction (XRD) and X-ray photoelectron spectroscopy (XPS). Comparison with MEGO indicates that the (002) peak of a-MEGO has a significantly reduced intensity and is dramatically broadened. These results are consistent with the observations from HRTEM, which indicate that a-MEGO is composed of predominantly single carbon sheets: thus a strong decrease in the (002) peak would be expected. A significant increase in the low-angle scatter from a-MEGO vs. MEGO is also noted, which is consistent with the presence of a high density of pores. In the XPS C1s spectrum of MEGO shown in Fig. 2 B, the tail between 286 and $290 \mathrm{eV}$ is due to $\mathrm{C}-\mathrm{O}$ groups and energy loss "shake-up" features (26). These oxygen containing groups were strongly suppressed after activation with two new peaks appearing between 292 and $296 \mathrm{eV}$ in the a-MEGO sample that were assigned as $\mathrm{K} 2 p$ peaks. The $\mathrm{K} 2 p$ peaks ( $<2$ at. $\%$ as determined from XPS) in a-MEGO are due to potassium residue, primarily as $\mathrm{K}_{2} \mathrm{CO}_{3}$ with a small amount of $\mathrm{KOH}$. Quantification of the amount of $\mathrm{sp}^{2}$-bonding can be determined by measuring the ratio between $\pi^{*}$ bonding and $\pi^{*}+\delta^{*}$ bonding using electron energy loss spectroscopy (EELS) (27). Figure 2C presents a comparison of the carbon $\mathrm{K}$ near edge structure for a-MEGO and graphite of equivalent thickness. With the assumption that the $\mathrm{sp}^{2}$ bonding in the graphite reference spectra is $100 \%$, the aMEGO was found to have $98 \%( \pm 2 \%) \mathrm{sp}^{2}$ bonding. Complementary measurements were also made by XPS reaching similar conclusions (further details of the fitting procedures can be found in fig. S4). Micro Raman spectroscopy (fig. S5 A) and Fourier transform infrared spectroscopy (fig. S5 B) are provided for completeness but did not supply additional insights about a-MEGO beyond the other methods of analysis discussed here.

State of the art surface and pore size characterization of the a-MEGO was performed by coupling high resolution nitrogen $(77.4 \mathrm{~K})$ and argon $(87.3 \mathrm{~K})$ adsorption/desorption experiments with advanced methods based on density functional theory (28). In addition, $\mathrm{CO}_{2}$ adsorption at $273.2 \mathrm{~K}$ 
has been performed to assess the ultramicropores (i.e., pores of width $<1 \mathrm{~nm}$ ). Figure $3 \mathrm{~A}$ shows these isotherms which reveal the details of the low-pressure region where micropore filling occurs, as well as the linear plot of the argon and nitrogen isotherms that reveal pore condensation and type $\mathrm{H} 2$ hysteresis (according to the IUPAC classification) (29), for the a-MEGO sample, which is indicative of an interconnected pore system exhibiting constrictions $(29,30)$. For comparison, nitrogen adsorption on a MEGO control sample is also shown in fig. S6 and it is evident that the the pore volume of MEGO is much smaller than that of a-MEGO; the small overall pore volume should be caused by the platelet like structure in MEGO, i.e., are likely just the gap between platelets (20). In contrast, the pores in a-MEGO have a welldefined micro-mesopore size distribution as shown in Fig. 3 $B$ with a huge increase in pore volume (up to $2.14 \mathrm{~cm}^{3} / \mathrm{g}$ ) relative to MEGO. The a-MEGO had a high nitrogen BET SSA of $\sim 3100 \mathrm{~m}^{2} / \mathrm{g}$ (calculated in the linear relative pressure range from 0.1 to 0.3 ). Within this context it should be noted that in a strict sense the BET method is not applicable to microporous solids, and hence the obtained BET surface area should be considered as an apparent or equivalent area only. Figure 3B displays the results of the cumulative pore volume and pore size analysis from $\mathrm{CO}_{2}$ and nitrogen adsorption by applying a hybrid NLDFT kernel assuming a slit pore geometry for the micropores and a cylindrical pore geometry for the mesopores which appears (although oversimplified) to be a reasonable assumption also with regard to the SEM/STEM/TEM results. The obtained pore size/volume distribution indicates that this carbon sample is quite unique because of the existence of well-defined micro- and mesopores. The presence of ultramicropores is seen from the $\mathrm{CO}_{2}$ data, and the analysis of the nitrogen adsorption data reveals the presence of micropores in the $\sim 1 \mathrm{~nm}$ size range as well as narrow mesopores centered around $4 \mathrm{~nm}$ in size. The latter is in good agreement with the bimodal distribution of pore sizes observed by high-resolution electron microscopy images. A 'quenched solid density functional theory'

(QSDFT), which quantitatively accounts for the surface roughness (31) has been also used to obtain the pore size distribution of a-MEGO from the nitrogen and argon adsorption data, as shown in fig. S7. It should be also noted that the pore size distribution curves obtained from argon and nitrogen agree very well.

Using best practice methods for determining an electrode material's performance for supercapacitors (32), we constructed and measured the performance of two-electrode symmetrical supercapacitor cells based on a-MEGO (SSA $2400 \mathrm{~m}^{2} / \mathrm{g}$ ) and 1-Butyl-3-methyl-imidazolium tetrafluoroborate $\left(\mathrm{BMIM} \mathrm{BF}_{4}\right) / \mathrm{AN}$ electrolyte, as shown in Fig. 4. The cyclic voltammetry (CV) testing (Fig. $4 \mathrm{~A}$ ) shows rectangular curves from 0 to $3.5 \mathrm{~V}$ over a wide range of voltage scan rates. Figure $4 \mathrm{~B}$ shows the galvanostatic charge/discharge curves at three current densities. The specific capacitance was calculated from the discharge curves with values of 165,166 , and $166 \mathrm{~F} / \mathrm{g}$ obtained at current densities of $1.4,2.8$, and $5.7 \mathrm{~A} / \mathrm{g}$, respectively. The corresponding volumetric capacitance is $\sim 60 \mathrm{~F} / \mathrm{cm}^{3}$. The voltage drop at the initiation of the discharge is $0.034 \mathrm{~V}$ (for the current density of $1.4 \mathrm{~A} / \mathrm{g}$ ), suggesting a very low ESR in the test cell. A frequency response analysis (FRA) of the frequency range from $500 \mathrm{kHz}$ to $5 \mathrm{mHz}$ yields the Nyquist plot shown in Fig. 4C. The plot features a vertical curve, indicating a nearly ideal capacitive behavior of the cell. From the magnified data in the high frequency range (Fig. 4C inset), a transition between the RC semicircle and the migration of electrolyte was observed at a frequency of about $382 \mathrm{~Hz}$, corresponding to a resistance of $2.45 \Omega$. The diffusion of electrolyte ions stopped at about $3 \mathrm{~Hz}$ and thereafter the whole capacitance is reached (33). The voltage drop at the beginning of discharge curves was used to estimate the internal resistance. An ESR of $3.2 \Omega$ was obtained from a-MEGO in the BMIM $\mathrm{BF}_{4} / \mathrm{AN}$ electrolyte. Based on a series RC model, the capacitance from the FRA data as a function of frequency is shown in Fig. 4 D. The capacitance decreases sharply at about $4 \mathrm{~Hz}$ and remains $0.035 \mathrm{~F}$ at $10 \mathrm{~Hz}$. Supercapacitor performance of the aMEGO (SSA $\sim 3100 \mathrm{~m}^{2} / \mathrm{g}$ ) in the TEA $\mathrm{BF}_{4} / \mathrm{AN}$ electrolyte was also measured and yielded a specific capacitance of above $150 \mathrm{~F} / \mathrm{g}$ as obtained from the discharge curve with a constant current of $0.8 \mathrm{~A} / \mathrm{g}$, with an ESR of $4.6 \Omega$ (fig. S8). One other carbon ( $\mathrm{KOH}$ activated $\mathrm{CDCs}$ ) using the same ANbased electrolyte with comparable specific capacitance values has been reported (34). A-MEGO has the highest gravimetric capacitance in organic electrolyte reported to date for any carbons derived from graphene-based materials.

Values for energy and power density were estimated based on the supercapacitor measurements in the BMIM BF $4 / \mathrm{AN}$ electrolyte. Using the specific capacitance value of $166 \mathrm{~F} / \mathrm{g}$ (from the discharge curve with a constant current of $5.7 \mathrm{~A} / \mathrm{g}$ ) and working voltage of $3.5 \mathrm{~V}$, the energy density is $\sim 70$ $\mathrm{Wh} / \mathrm{kg}$ for the a-MEGO in the cell. Based on a weight ratio of $30 \%$ for the active electrode material in a packaged supercapacitor device, typical for large scale AC based supercapacitors, a practical energy density of above 20 $\mathrm{Wh} / \mathrm{kg}$ for a packaged device is expected. This is 4 times higher than existing AC-based supercapacitors, 2 times higher than that reported for carbon-oxide hybrid electrochemical devices (2), and nearly equal to the energy density of lead acid batteries. At the same current density $(5.7 \mathrm{~A} / \mathrm{g})$, the power density is also very high at $\sim 250 \mathrm{~kW} / \mathrm{kg}$, as estimated by using the voltage drop and ESR obtained from the discharge curve. For a packaged cell, the power density of $75 \mathrm{~kW} / \mathrm{kg}$ is one order higher than the values from 
commercial carbon supercapacitors that have energy density values of $4-5 \mathrm{Wh} / \mathrm{kg}$ (2). This material is also very stable. After 10,000 constant current charge/discharge cycles at a current density of $2.5 \mathrm{~A} / \mathrm{g}$ in neat $\mathrm{BMIM} \mathrm{BF}_{4}$ electrolyte (fig. S9), $97 \%$ of its capacitance was retained. We reasoned that this carbon might perform even better with smaller diameter ions (35). Indeed with neat EMIM TFSI as electrolyte (fig. S10), the measured gravimetric capacitance of a-MEGO $\left(\mathrm{SSA} \sim 3100 \mathrm{~m}^{2} / \mathrm{g}\right.$ ) at $3.5 \mathrm{~V}$ and a current density of $0.7 \mathrm{~A} / \mathrm{g}$ is $200 \mathrm{~F} / \mathrm{g}$, with an ESR of $8.6 \Omega$. However, the curves in fig. $\mathrm{S} 10$ are not as ideal as those from a-MEGO in either BMIM $\mathrm{BF}_{4} / \mathrm{AN}$ or TEA BF$/ / \mathrm{AN}$ electrolyte. We also note that other carbons (e.g. CDCs) using the EMIM TSFI electrolyte have been reported with comparable performance values although the measurements were performed at elevated temperature $\left(60{ }^{\circ} \mathrm{C}\right)(36)$.

The high powder conductivity of $\sim 500 \mathrm{~S} / \mathrm{m}$, in addition to a $\mathrm{C} / \mathrm{O}$ atomic ratio of up to $\sim 35$ and the very low $\mathrm{H}$ content, along with the essential absence of dangling bonds in the aMEGO suggest that it has a high content of $\mathrm{sp}^{2}$-bonded carbon and very few edge atoms, for the samples with SSA of above $2500 \mathrm{~m}^{2} / \mathrm{g}$. This along with the SEM, (S)TEM, EELS, EPR, XPS and XRD, and adsorption isotherm data, thus support a highly porous carbon comprised almost entirely of single sheets of $\mathrm{sp}^{2}$-bonded carbon. This suggests that a significant fraction of 'negative curvature carbon' $(37,38)$ could be present.

The excellent performance obtained for various electrolytes opens the possibility to engineer supercapacitor electrodes based on this form of carbon to target a wide range of applications such as high energy, high power, or low cost. Unlike other carbon materials, no special substrates or transfer procedures are required for synthesis. For supercapacitor manufacturing, this material can be treated the same as current commercial activated carbons. Electrodes used for testing were of the same thickness used in commercial cells and testing was performed using commercial collectors, separators, binders, and electrolytes. As previously stated, the processes used to synthesize this carbon electrode material are readily scalable to industrial levels. For example, we have demonstrated that this simple activation process also can be applied to TEGO (figs. S11 and $\mathrm{S} 12$ ), which is already being manufactured in ton quantities (13). By use of this type of simple activation process already commercially demonstrated for ACs, scaled a-MEGO and aTEGO production for advanced energy/power electrochemical electrical energy storage devices might be realized in a short period.

\section{References and Notes}

1. J. R. Miller, P. Simon, Science 321, 651 (2008).

2. A. Burke, Electrochimica Acta 53, 1083 (2007).
3. J. A. Fernandez et al., Journal of Power Sources 175, 675 (2008).

4. P. Simon, Y. Gogotsi, Nature Materials 7, 845 (2008).

5. J. Chmiola et al., Science 313, 1760 (2006).

6. N. L. Wu, Materials Chemistry and Physics 75, 6 (2002).

7. T. Brezesinski, J. Wang, S. H. Tolbert, B. Dunn, Nature Materials 9, 146 (2010).

8. A. Rudge, J. Davey, I. Raistrick, S. Gottesfeld, J. P. Ferraris, Journal of Power Sources 47, 89 (1994).

9. M. Toupin, T. Brousse, D. Belanger, Chemistry of Materials 16, 3184 (2004).

10. A. Izadi-Najafabadi et al., Advanced Materials 22, E235 (2010).

11. D. N. Futaba et al., Nature Materials 5, 987 (2006).

12. Y. Zhu et al., Advanced Materials 22, 3906 (2010).

13. M. Segal, Nat Nano 4, 612 (2009).

14. M. D. Stoller, S. J. Park, Y. W. Zhu, J. H. An, R. S. Ruoff, Nano Letters 8, 3498 (2008).

15. Y. Wang et al., The Journal of Physical Chemistry C 113, 13103 (2009).

16. W. Lv et al., ACS Nano 3, 3730 (2009).

17. Y. Zhu et al., ACS Nano 4, 1227 (2010).

18. S. R. C. Vivekchand, C. S. Rout, K. S. Subrahmanyam,

A. Govindaraj, C. N. R. Rao, Journal of Chemical

Sciences 120, 9 (2008).

19. J. R. Miller, R. A. Outlaw, B. C. Holloway, Science 329, 1637 (2010).

20. Y. Zhu et al., Carbon 48, 2118 (2010).

21. H. Marsh, F. Rodriguez-Reinoso, Actiaved Carbon. (Elsevier, 2006).

22. E. Raymundo-Pinero et al., Carbon 43, 786 (2005).

23. V. Barranco et al., The Journal of Physical Chemistry C 114, 10302 (2010).

24. T. Liu, T. V. Sreekumar, S. Kumar, R. H. Hauge, R. E. Smalley, Carbon 41, 2440 (2003).

25. M. A. Lillo-Rodenas, D. Cazorla-Amoros, A. LinaresSolano, Carbon 41, 267 (2003).

26. J. A. Leiro, M. H. Heinonen, T. Laiho, I. G. Batirev, Journal of Electron Spectroscopy and Related Phenomena 128, 205 (2003).

27. S. D. Berger, D. R. McKenzie, P. J. Martin, Phil. Mag. Lett. 57, 285 (1988).

28. P. I. Ravikovitch, A. Vishnyakov, R. Russo, A. V. Neimark, Langmuir 16, 2311 (2000).

29. K. S. W. Sing et al., Pure Appl. Chem. 57, 603 (1985).

30. J. C. Groen, L. A. A. Peffer, J. Perez-Ramirez, Microporous and Mesoporous Materials 60, 1 (2003).

31. A. V. Neimark, Y. Lin, P. I. Ravikovitch, M. Thommes, Carbon 47, 1617 (2009).

32. M. D. Stoller, R. S. Ruoff, Energy \& Environmental Science 3, 1294 (2010). 
33. P. L. Taberna, P. Simon, J. F. Fauvarque, Journal of the Electrochemical Society 150, A292 (2003).

34. C. Portet, M. A. Lillo-Rodenas, A. Linares-Solano, Y. Gogotsi, Physical Chemistry Chemical Physics 11, 4943 (2009).

35. C. O. Ania, J. Pernak, F. Stefaniak, E. Raymundo-Pinero, F. Beguin, Carbon 44, 3126 (2006).

36. C. Largeot et al., Journal of the American Chemical Society 130, 2730 (2008).

37. S. J. Townsend, T. J. Lenosky, D. A. Muller, C. S. Nichols, V. Elser, Physical Review Letters 69, 921 (1992).

38. H. Terrones, A. L. Mackay, Progress in Crystal Growth and Characterization of Materials 34, 25 (1997).

Acknowledgments: We appreciate funding support from NSF, DOE, and the Institute of Applied Technology. The work at the Center for Functional Nanomaterials at the Brookhaven National Laboratory is supported under USDOE contract DE-AC02-98CH10886. We thank P. Stephens for XRD data collection, which is supported by US-DOE under contract DE-AC02-98CH10886 and P. Ercius for data collection on the TEAM instrument, supported by US-DOE contract DE-AC02-05CH11231. We thank M. Nilges for help with EPR. We thank J. Potts for providing graphite oxide samples. We appreciate use of equipment in K. Johnston's lab. RMW and AP acknowledge the partial support of the GRC-NRI SWAN Center for the XPS data collection and analysis.

\section{Supporting Online Material}

Www.sciencemag.org/cgi/content/full/science.1200770/DC1

SOM Text

Figs. S1 to S12

References

Movie S1

22 November 2010; accepted 2 May 2011

Published online 12 May 2011; 10.1126/science. 1200770

Fig. 1 (A) Schematic showing the microwave exfoliation/reduction of GO and the following chemical activation of MEGO with $\mathrm{KOH}$ that creates pores while retaining high electrical conductivity. (B) Low magnification SEM image of a 3D a-MEGO chunk that demonstrates its porous morphology. (C) High-resolution SEM image of a different sample region. (D) ADF-STEM image of the same area as (C), acquired simultaneously. As seen, a-MEGO contains micro- and mesopores with a distribution of sizes between 1-10 nm. (E) High-resolution phase contrast electron micrograph of the thin edge of an a-MEGO chunk, taken at $80 \mathrm{kV}$. There is a variation in focus across the image due to the sloped nature of the sample and changes in sample thickness. The image shows the presence of a dense network of nanometer-scale pores surrounded by highly curved, predominantly single layer carbon. (F) Exit wave reconstructed HRTEM image from the edge of a-MEGO. The in-plane carbon atoms are clearly resolved, and a variety of nmembered carbon rings can be seen. Significant curvature of the single carbon sheets is visible, with the in-plane crystallinity being preserved.

Fig. 2 Characterization of the a-MEGO material (SSA $\sim 2520$ $\mathrm{m}^{2} / \mathrm{g}$ ) with MEGO or graphite as a control. (A) Synchrotron Powder XRD pattern (plotted as $\mathrm{Cu} \mathrm{K} \alpha$ ). FWHM of the (100) peak is $\sim 2$ degrees for both samples, indicating average inplane crystal size of $\sim 5 \mathrm{~nm}$ by the Scherrer equation; the disappearance of the (002) in a-MEGO indicates that there are essentially no interlayer correlations of the carbon sheets.

(B) XPS C $1 s$ spectra with $\mathrm{K} 2 p$ region in the inset. (C) EELS spectra from a-MEGO and graphite. Quantification of the near edge structure indicates that the a-MEGO has $98 \%$ $( \pm 2 \%) \mathrm{sp}^{2}$ bonding.

Fig. 3 Gas adsorption/desorption analysis of an a-MEGO sample (SSA $\sim 3100 \mathrm{~m}^{2} / \mathrm{g}$ ). (A) High resolution, low pressure $\mathrm{N}_{2}(77.4 \mathrm{~K})$ and $\mathrm{Ar}(87.3 \mathrm{~K})$ isotherms. Inset shows the $\mathrm{CO}_{2}$ (273.2 K) isotherm. (B) Cumulative pore volume and (inset) pore size distribution for $\mathrm{N}_{2}$ (calculated using a slit/cylindrical NLDFT model) and $\mathrm{CO}_{2}$ (calculated using a slit pore NLDFT model).

Fig. 4 Supercapacitor performance of a-MEGO (SSA 2400 $\mathrm{m}^{2} / \mathrm{g}$ ) in the BMIM BF $4 / \mathrm{AN}$ electrolyte. (A) CV curves for different scan rates. Rectangular shapes indicate the capacitive behavior. (B) Galvanostatic charge/discharge curves of a-MEGO based supercapacitor under different constant currents. (C) Nyquist plot, showing the imaginary part versus the real part of impedance. Inset shows the data at high frequency ranges, with frequency values corresponding to the transition of the curves marked. (D) Frequency response of the gravimetric capacitance of the a-MEGO supercapacitor. Capacitances of 35 and $\sim 8.8 \mathrm{mF}$ remain at the frequencies of 10 and $100 \mathrm{~Hz}$, respectively. 

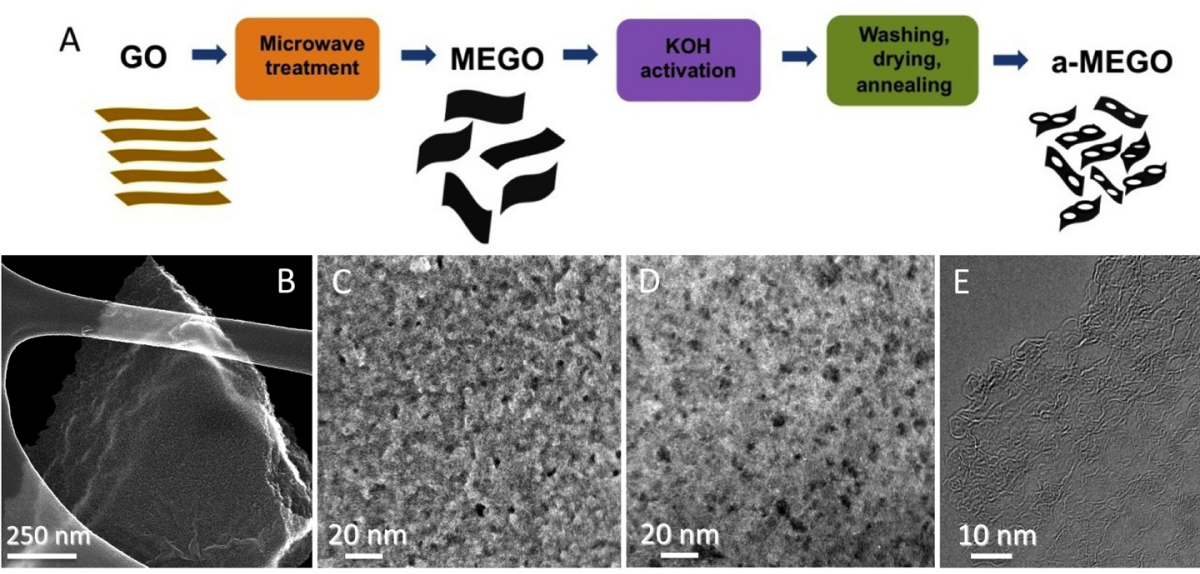

F

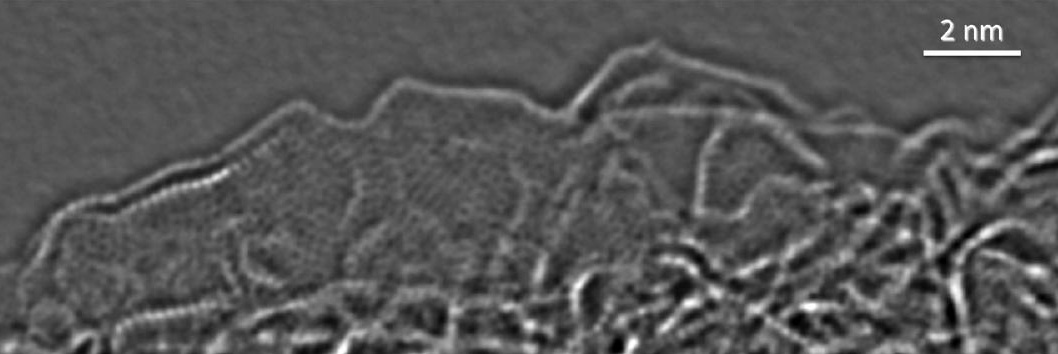


